\title{
Perfil de limiares ventilatórios durante o exercício e o consumo de oxigênio de pico verificado em jogadoras de futebol
}

\author{
Paulo Roberto Santos Silva1, Adilson Andrade ${ }^{2}$, Wilson Oliveira Riça ${ }^{3}$, Ana Maria Visconti ${ }^{4}$, \\ Fernando Miele da Ponte ${ }^{5}$, Albertina Fontana Rosa ${ }^{6}$, Solange Basílio da Costa ${ }^{6}$, \\ Carla Dal Maso Nunes Roxo ${ }^{7}$, Gilberto Silva Machado ${ }^{7}$ e Jorge Mendes de Sousa ${ }^{8}$
}

Seção de Fisiologia do Centro de Medicina Integrada da Associação Portuguesa de Desportos, SP, Brasil

\section{RESUMO}

Foram avaliadas 37 jogadoras de futebol, com média de idade de $21,5 \pm 5,9$ anos, peso de $57,0 \pm 8,3 \mathrm{~kg}$, estatura de $161 \pm 7 \mathrm{~cm}$ e índice de massa corpórea de $21,8 \pm 2,1 \mathrm{~kg} / \mathrm{m}^{2}$. Todas as atletas foram submetidas à avaliação espirométrica e metabólica, por meio de um sistema computadorizado de análise de gases expirados (modelo Vmax 29c, SensorMedics, EUA). A resposta cardiovascular foi verificada por meio de um eletrocardiógrafo computadorizado (modelo 6.4, HeartWa$r e$, BRA). A capacidade física máxima foi avaliada por meio de teste de esforço realizado em esteira rolante (modelo $A T L$ 10.100, Inbramed, BRA) utilizando-se protocolo escalonado contínuo. Os seguintes parâmetros e os resultados encontrados foram: no limiar ventilatório $1\left(\mathrm{LV}_{1}\right): \mathrm{VO}_{2}=30,5 \pm 3,7 \mathrm{ml}$ $\mathrm{O}_{2} \cdot \mathrm{kg}^{-1} \cdot \mathrm{min}^{-1} ; \% \mathrm{VO}_{2}=64 \pm 7 \%$; velocidade de corrida $=8,1$ $\pm 0,3 \mathrm{~km} \cdot \mathrm{h}^{-1} ; \mathrm{FC}=154 \pm 9 \mathrm{bpm}$. No limiar ventilatório $2\left(\mathrm{LV}_{2}\right)$ : $\mathrm{VO}_{2}=40,9 \pm 4,5 \mathrm{ml} \mathrm{O}_{2} \cdot \mathrm{kg}^{-1} \cdot \mathrm{min}^{-1} ; \% \mathrm{VO}_{2}=85,7 \pm 4,9 \%$; velocidade de corrida $=11,4 \pm 1,1 \mathrm{~km} \cdot \mathrm{h}^{-1} ; \mathrm{FC}=179 \pm 7 \mathrm{bpm}$. O $\mathrm{VO}_{2}$ de pico foi de $47,4 \pm 4,1 \mathrm{ml} \mathrm{O} \cdot \mathrm{kg}^{-1} \cdot \mathrm{min}^{-1}$. Conclusão: a verificação de limiares ventilatórios e a potência aeróbia em jogadoras de futebol são parâmetros fisiológicos de grande importância, pois permitem ampliar suas aplicações práticas,

\footnotetext{
1. Fisiologista.

2. Fisicultor.

3. Técnico de Futebol Feminino.

4. Clínico.

5. Ortopedista.

6. Odontologista.

7. Fisioterapeuta.

8. Vice-Presidente de Medicina da Associação Portuguesa de Desportos.
}

Endereço para correspondência:

Paulo Roberto Santos Silva

Centro de Medicina Integrada - Seção de Fisiologia

Rua Comendador Nestor Pereira, 33 - Canindé

03034-070 - São Paulo, SP

Tel. (011) 3315-0400 - R. 228

Fax (011) 228-2001 qualificando, controlando e desenvolvendo de modo mais adequado e objetivo o treinamento físico dessas atletas.

Palavras-chave: Limiares ventilatórios. Consumo de oxigênio de pico. Futebolistas femininas. Medicina esportiva.

\section{ABSTRACT \\ Ventilatory threshold profile during exercise and peak oxy- gen uptake verified in female soccer players}

Thirty-seven female soccer players aged $21.5 \pm 5.9$, weight $57.0 \pm 8.3 \mathrm{~kg}$, height $161 \pm 7 \mathrm{~cm}$ and body mass index $21.8 \pm$ $2.1 \mathrm{~kg} / \mathrm{m}^{2}$ were submitted to spirometric and metabolic evaluation by a computerized analysis system of expired gases (model Vmax 29c, SensorMedics, EUA). Cardiovascular response was recorded by means of a computerized ECG (model 6.4, HeartWare, BRA). Maximum physical capacity was evaluated by a treadmill test (model ATL 10.100, Inbramed, BRA), using incremental continuous protocol. The parameters and results were: in ventilatory threshold $1\left(V T_{1}\right): V O_{2}=30.5 \pm 3.7$ $\mathrm{ml} \mathrm{O}_{2} \cdot \mathrm{kg}^{-1} \cdot \mathrm{min}^{-1} ; \% \mathrm{VO}_{2}=64 \pm 7 \%$; running velocity $=8.1 \pm$ $0.3 \mathrm{~km} \cdot \mathrm{h}^{-1} ; \mathrm{HR}=154 \pm 9 \mathrm{bpm}$; in ventilatory threshold 2 $\left(V T_{2}\right): V O_{2}=40.9 \pm 4.5 \mathrm{ml} \mathrm{O} \cdot \mathrm{kg}^{-1} \cdot \mathrm{min}^{-1} ; \% \mathrm{VO}_{2}=85.7 \pm$ $4.9 \%$; running velocity $=11.4 \pm 1.1 \mathrm{~km} \cdot \mathrm{h}^{-1} ; \mathrm{HR}=179 \pm 7$ bpm. The peak $\mathrm{VO}_{2}$ was $47.4 \pm 4.1 \mathrm{ml} \mathrm{O}_{2} . \mathrm{kg}^{-1}$. $\mathrm{min}^{-1}$. In conclusion, the ventilatory thresholds and the aerobic power verified in female soccer players are physiological parameters of great importance, as they make it possible to enlarge their practical application, qualifying, controlling and developing the physical training of such athletes in a more practical and objective manner.

Key words: Ventilatory thresholds. Peak oxygen uptake. Female soccer players. Sports medicine.

\section{INTRODUÇÃO}

O futebol feminino para o mundo ainda não é uma realidade. Tradicionalmente, ele tem sido praticado por homens, o que de certo modo tem inibido a coragem de muitas mulhe- 
res, pois ainda há um clima de hostilidade por muitos, principalmente do sexo masculino.

Apesar das dificuldades, a história relata que, desde 1900, as mulheres já jogavam futebol na Inglaterra. Em 1993, a Liga Nacional Inglesa tinha 11.000 jogadoras registradas com mais de 24 equipes.

Em 1988, no Japão, 9.647 futebolistas foram inscritas na Associação Japonesa de Futebol, com um total de 470 equipes $^{1}$.

Para promover o status dessa modalidade praticada por mulheres, a Fédération Internationale de Football Association (FIFA), órgão máximo e organizador desse esporte, realizou o primeiro campeonato mundial em 1991, nos Estados Unidos da América.

No Brasil o futebol feminino vem crescendo em importância, número de praticantes e desenvolvimento científico. Entretanto, há ainda escassez muito grande de publicações sobre parâmetros fisiológicos verificados em jogadoras de futebol no exterior e, principalmente, em nosso país.

Sabe-se que limiares ventilatórios e a potência aeróbia são parâmetros fisiológicos considerados importantes preditores para definir a aptidão de um indivíduo para tolerar exercício de intensidade submáxima e de longa duração, como é o futebol.

Portanto, a verificação periódica desses índices funcionais, além de controlar melhor a intensidade do treinamento, permite desenvolver de maneira mais precisa o objetivo-alvo a ser atingido pelos atletas.

O propósito do presente estudo foi mostrar o perfil dos limiares ventilatórios e o consumo de oxigênio de pico, verificados em jogadoras de futebol, participantes de equipes que disputam os campeonatos oficiais em nosso país.

\section{MATERIAL E MÉTODOS}

Foram avaliadas 37 jogadoras de futebol, com média de idade de 21,5 $\pm 5,9$ anos (14-34), peso de 57,0 $\pm 8,3 \mathrm{~kg}$ (45$79)$, estatura de $161 \pm 7 \mathrm{~cm}$ (146-179), índice de massa corpórea (IMC) de 21,8 $\pm 2,1 \mathrm{~kg} \cdot \mathrm{m}^{2}(18,8-27,3)$. Suas características cardiovasculares em repouso estão listadas na tabela 1. As condições meteorológicas durante as realizações dos testes foram as seguintes: temperatura ambiente de $19,2 \pm 1,6^{\circ} \mathrm{C}(16$ 22), pressão barométrica de $706,7 \pm 2,7 \mathrm{mmHg}$ (703-710) e umidade relativa percentual do ar de $66,6 \pm 10,5 \%$ (45-76) [tabela 1].

Previamente à avaliação ergométrica, todas as atletas foram submetidas a eletrocardiograma (ECG), (modelo 6.4, Heart-Ware, BRA) em repouso e durante teste de esforço por meio da monitoração de 12 derivações, segundo posição preconizada por Mason \& Likar, com modificação da derivação $\left(\mathrm{D}_{1}\right.$ para $\left.\mathrm{MC}_{5}\right)$ e registradas por impressora a jato de tinta (modelo 680c, HP DeskJet). A pressão arterial (PA) foi medida por método auscultatório indireto, utilizando-se esfigmomanômetro aneróide (Tycos, EUA).
A ventilação pulmonar $\left(\mathrm{V}_{\mathrm{E} \text { в втPS }}\right)$, o consumo de oxigênio $\left(\mathrm{VO}_{2 \text { STPD }}\right)$, a produção de dióxido de carbono $\left(\mathrm{VCO}_{2 \text { STPD }}\right)$ e a razão de troca respiratória (RER) foram calculados a partir de valores medidos por um sistema computadorizado de análise de troca gasosa (respiração-a-respiração) (modelo Vmax 29c, SensorMedics, EUA). O volume ventilatório foi medido por um sensor de fluxo de massa (SensorMedics, EUA).

A calibração foi feita antes da realização de cada teste com uma seringa de três litros, para ser empregado fator de correção que determinará o volume respiratório. As frações expiradas de oxigênio $\left(\mathrm{F}_{\mathrm{E}} \mathrm{O}_{2}\right)$ foram medidas por um sensor paramagnético de resposta rápida e elevada precisão (SensorMedics, EUA) e as frações expiradas de dióxido de carbono $\left(\mathrm{F}_{\mathrm{E}} \mathrm{CO}_{2}\right)$ pelo princípio do infravermelho.

A calibração dos analisadores do equipamento foi feita antes e imediatamente após a realização de cada teste com mistura gasosa conhecida de $\mathrm{O}_{2}(12 \%$ e $26 \%), \mathrm{CO}_{2}(4 \%)$ e balanceada com nitrogênio $\left(\mathrm{N}_{2}\right)$. As variáveis ventilatórias foram registradas instantaneamente e posteriormente calculadas para o tempo médio de 10 segundos.

A determinação da capacidade física máxima foi verificada realizando-se um teste de esforço em esteira rolante (modelo ATL-10.100, Inbramed, BRA) de velocidade $\left(\mathrm{km} . \mathrm{h}^{-1}\right)$ e inclinação (\%) variáveis, utilizando-se protocolo escalonado contínuo e inclinação fixa de $3 \%$.

Nesse protocolo, a atleta ficou dois minutos em repouso, foi aquecida por quatro minutos nas velocidades de 4, 5, $6 \mathrm{e}$ $7 \mathrm{~km} \cdot \mathrm{h}^{-1}$ durante um minuto em cada velocidade. Posteriormente, iniciou-se o teste com $8 \mathrm{~km} \cdot \mathrm{h}^{-1}$ e incrementos de $1 \mathrm{~km}$. $\mathrm{h}^{-1}$ a cada dois minutos até a exaustão da atleta. A fase de recuperação durou quatro minutos e foi realizada com velocidades controladas a 60, 50, 40 e 30\% da velocidade máxima atingida pela atleta no teste. A percepção subjetiva ao esforço foi verificada em cada estágio do teste pela escala linear gradual de 15 pontos (6 a 20) de Borg².

Os limiares ventilatórios $1\left(\mathrm{LV}_{1}\right)$ e $2\left(\mathrm{LV}_{2}\right)$ foram determinados, utilizando-se os seguintes critérios: $\mathrm{LV}_{1}-1^{\mathrm{O}}$ ) menor valor do equivalente ventilatório de oxigênio $\left(\mathrm{V}_{\mathrm{E}} \cdot \mathrm{VO}_{2}{ }^{-1}\right)$ e $\left.2^{\mathrm{o}}\right)$ menor valor da fração expirada de oxigênio $\left(\mathrm{F}_{\mathrm{E}} \mathrm{O}_{2}\right)$, ambos seguidos de inflexão e crescimento ao longo do teste. $\mathrm{LV}_{2}$ 19) menor valor do equivalente ventilatório de dióxido de carbono $\left(\mathrm{V}_{\mathrm{E}} \cdot \mathrm{VCO}_{2}^{-1}\right)$ seguido de inflexão e incremento posterior e $2^{\circ}$ ) maior valor da fração expirada de dióxido de carbono $\left(\mathrm{F}_{\mathrm{E}} \mathrm{CO}_{2}\right)$ seguido de diminuição até o final do teste. Ambos os limiares foram verificados em exercício de intensidade progressiva ${ }^{3}$.

A análise dos dados foi realizada calculando-se a média, o desvio-padrão e as variações mínima e máxima dos parâmetros avaliados ${ }^{4}$.

\section{RESULTADOS}

Os resultados deste estudo estão listados nas tabelas 1, 2 e 3 . 


\begin{tabular}{|c|c|c|c|c|c|c|}
\hline \multicolumn{7}{|c|}{$\begin{array}{l}\text { TABELA } 1 \\
\text { Valores médios, mínimos e máximos das características físicas das jogadoras }(n=37) \\
\text { de futebol e as condições meteorológicas durante a realização dos testes de esforço }\end{array}$} \\
\hline $\begin{array}{l}\text { Idade } \\
\text { (anos) }\end{array}$ & $\begin{array}{l}\text { Peso } \\
\text { (kg) }\end{array}$ & $\begin{array}{c}\text { Estatura } \\
(\mathbf{c m})\end{array}$ & $\begin{array}{c}\text { IMC } \\
\left(\mathbf{k g} / \mathbf{m}^{2}\right)\end{array}$ & $\begin{array}{l}\text { Temperatura } \\
\text { ambiente } \\
\left({ }^{\circ} \mathrm{C}\right)\end{array}$ & $\begin{array}{l}\text { Pressão } \\
\text { barométrica } \\
(\mathbf{m m H g})\end{array}$ & $\begin{array}{l}\text { Umidade } \\
\text { relativa do ar } \\
\text { (\%) }\end{array}$ \\
\hline $\begin{array}{c}21,5 \\
\pm 5,9 \\
(14-34)\end{array}$ & $\begin{array}{r}57,0 \\
\pm 8,3 \\
(45-79)\end{array}$ & $\begin{array}{l}161 \\
\quad \pm 7 \\
(146-179)\end{array}$ & $\begin{array}{c}21,8 \\
\pm 2,1 \\
(18,8-27,3)\end{array}$ & $\begin{array}{c}19,2 \\
\pm 1,6 \\
(16-22)\end{array}$ & $\begin{array}{c}706,7 \\
\pm 2,7 \\
(703-710)\end{array}$ & $\begin{array}{r}66,6 \\
\pm 10,5 \\
(45-76)\end{array}$ \\
\hline
\end{tabular}

TABELA 2

\begin{tabular}{|c|c|c|c|}
\hline $\begin{array}{l}\text { Valores médi } \\
\text { e \%VO } \\
\text { ardíaca (bpm) } \\
\text { teste de es }\end{array}$ & $\begin{array}{l}\text { ínimos } \\
\text { cidade } \\
\text { miar ve } \\
\text { increm } \\
(n=3\end{array}$ & $\begin{array}{l}\text { iximos de } \mathrm{VO}_{2}( \\
\text { orrida }\left(\mathrm{km} \cdot \mathrm{h}^{-1}\right) \\
\text { tório } 1\left(\mathrm{LV}_{1}\right) \text {, det } \\
\text { I na esteira, em } \\
\text { futebol }\end{array}$ & $\begin{array}{l}\text { ü.min-1 } \\
\text { üência } \\
\text { nado em } \\
\text { doras }\end{array}$ \\
\hline $\begin{array}{c}\mathrm{VO}_{2} \\
\left.\mathrm{nl} \cdot \mathrm{kg}^{-1} \cdot \mathrm{min}^{-1}\right)\end{array}$ & $\begin{array}{l}\mathrm{VO}_{2} \\
(\%)\end{array}$ & $\begin{array}{l}\text { Veloc. comida } \\
\left(\mathrm{km} \cdot \mathrm{h}^{-1}\right)\end{array}$ & $\begin{array}{c}\mathrm{FC} \\
\text { (bpm) }\end{array}$ \\
\hline $\begin{array}{c}30,5 \\
\pm 3,7 \\
(24,1-37,8)\end{array}$ & $\begin{array}{c}64 \\
\pm 7 \\
(52-77)\end{array}$ & $\begin{array}{c}8,1 \\
\pm 0,3 \\
(8,0-9,0)\end{array}$ & $\begin{array}{c}154 \\
\pm 9 \\
(131-169)\end{array}$ \\
\hline
\end{tabular}

\section{DISCUSSÃO}

Desde o aparecimento na literatura especializada do termo limiar anaeróbio, verificado por técnica não-invasiva (análise de gases expirados) em 1964 e que foi nomeado por Wasserman \& McIlroy ${ }^{5}$, ele sofreu uma série de modificações.

Contudo, ele tem sido aceito e considerado por diversos pesquisadores como um dos parâmetros-chave para definir a capacidade de um indivíduo tolerar exercício de intensidade submáxima. Entretanto, ele também tem sido motivo de controvérsia em vários aspectos, como: origem, conceito, terminologia e critérios de detecção.

A tendência mais recente da literatura fisiológica norteamericana é a utilização do termo limiar anaeróbio. Além disso, a grande maioria das publicações refere-se apenas a um limiar, ou seja, o primeiro limiar ou limiar ventilatório $1\left(\mathrm{LV}_{1}\right)$, chamado pelos norte-americanos de limiar anaeróbio ${ }^{6}$. Ao contrário, os europeus geralmente preocupam-se com o segundo limiar, ou seja, o limiar ventilatório $2\left(\mathrm{LV}_{2}\right)$.

Contudo, a orientação de Hughes et al., citados por Go$\mathrm{mes}^{7}$, sugere que, quando o limiar anaeróbio for determinado por medidas de gases expirados, o termo limiar ventilatório deve ser o preferido. Portanto, optamos pela denominação de $\mathrm{LV}_{1}$ e $\mathrm{LV}_{2}$, terminologia utilizada por Bhambhani \& Singh, do Canadás.

É importante lembrar que, em atletas, o mais importante deles para o desenvolvimento mais acentuado de sua aptidão

\begin{tabular}{|c|c|c|c|c|}
\hline \multicolumn{5}{|c|}{ 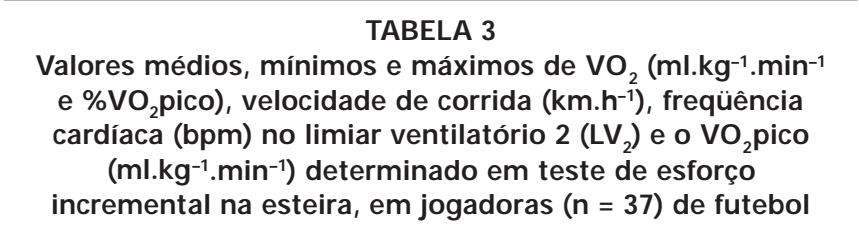 } \\
\hline 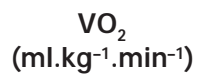 & $\begin{array}{l}\mathrm{VO}_{2} \\
\text { (\%) }\end{array}$ & $\begin{array}{l}\text { Veloc. corrida } \\
\left(\mathbf{k m} \cdot \mathrm{h}^{-1}\right)\end{array}$ & $\begin{array}{c}\text { FC } \\
\text { (bpm) }\end{array}$ & $\begin{array}{c}\text { VO }_{2} \text { pico } \\
\left(\mathrm{ml}^{\prime} \mathrm{kg}^{-1} \cdot \mathrm{min}^{-1}\right)\end{array}$ \\
\hline $\begin{array}{c}40,9 \\
\pm 4,5 \\
(31,8-46,3)\end{array}$ & $\begin{array}{c}85,7 \\
\pm 4,9 \\
(78-91)\end{array}$ & $\begin{array}{c}11,4 \\
\pm 1,1 \\
(9,0-14,0)\end{array}$ & $\begin{array}{c}179 \\
\pm 7 \\
(166-191)\end{array}$ & $\begin{array}{c}47,4 \\
\pm 4,1 \\
(40,7-57,6)\end{array}$ \\
\hline
\end{tabular}

aeróbia é treinar próximo do $\mathrm{LV}_{2}$, pois a eficiência metabólica neste ponto é maior.

A determinação dos limiares ventilatórios tem importante aplicação prática no controle da intensidade e na avaliação dos efeitos do treinamento. $\mathrm{O}$ consenso atual demonstra que um determinado parâmetro fisiológico isolado não contribui adequadamente para a compreensão dos mecanismos que interferem no rendimento físico do atleta.

Acredita-se que a detecção de fases metabólicas distintas ocorridas nos limiares ventilatórios pode identificar alterações fisiológicas que expliquem a capacidade para tolerar o exercício submáximo prolongado.

Portanto, a detecção desses momentos metabólicos é importante em qualquer atividade de longa duração, como é o futebol. Além disso, o referencial e a divisão dos limiares possibilitam o conhecimento de fases metabólicas distintas e com elas a possibilidade de determinar a intensidade de trabalho físico mais adequada para o objetivo pretendido.

Assim, as características metabólicas verificadas no $L_{1}$ são provenientes basicamente de atividade física realizada em baixa intensidade. Em nosso estudo, as futebolistas atingiram esse instante metabólico numa intensidade média de $64 \%$ do consumo de oxigênio de pico $\left(\mathrm{VO}_{2}\right.$ pico $)$ e com uma percepção subjetiva de cansaço, segundo escala de Borg, atingindo valor 9 (fácil). A média de resposta da freqüência cardíaca (FC) atingida no $\mathrm{LV}_{1}$ foi de $156 \mathrm{bpm}$, o que correspondeu a $81 \%$ da FC máxima atingida no final do teste. 
O LV 1 ocorre numa intensidade de exercício na qual o nível de ácido lático no sangue aumenta acima dos valores de repouso. Entretanto, nessa intensidade não se verifica diminuição significante do $\mathrm{pH}$ sanguíneo ${ }^{8,9}$.

Para eventos fisiológicos ocorridos nessa intensidade de exercício, a percentagem do consumo de oxigênio de pico verificada no $L V_{1}$ ficou levemente acima da faixa de variação do modelo hipotético proposto por Skinner \& McLellan ${ }^{10}$.

Exercício realizado nessa intensidade estimula pouco a produção e o acúmulo de ácido lático no músculo, pois tem participação predominante das fibras musculares do tipo I (oxidativas) ou de contração lenta (vermelhas) e que têm grande afinidade pelo oxigênio, pois são cercadas por maior número de capilares ${ }^{11}$.

Jorfeldt ${ }^{12}$ sugere que as fibras do tipo I, acionadas em exercício de baixa intensidade, continuamente extraem e oxidam o ácido lático do sangue proveniente das fibras do tipo IIb (brancas) que possuem elevado teor glicolítico anaeróbio.

Atividade física realizada utilizando-se a intensidade do exercício no $\mathrm{LV}_{1}$ é de grande utilidade no desenvolvimento da capilarização muscular após exercícios intensos, como em jogos e/ou treinamentos, em indivíduos que estão retornando de lesões musculares e que foram afastados de suas atividades por um longo período de tempo e/ou em indivíduos sedentários que querem iniciar um programa de treinamento físico.

Dessa forma, a resposta metabólica e ventilatória ao exercício em intensidade até o $\mathrm{LV}_{1}$, medida pela concentração de ácido lático sanguíneo, varia pouco em relação ao repouso, enquanto a ventilação se mantém estável ${ }^{13-15}$.

Portanto, em futebolistas, o referencial de intensidade do exercício realizado no $\mathrm{LV}_{1}$ é de grande valor prático para o fisicultor, que pode utilizá-lo de várias maneiras.

Ao contrário, as respostas metabólicas e ventilatórias verificadas em intensidade acima do $\mathrm{LV}_{2}$ são mais exacerbadas do que as verificadas no $\mathrm{LV}_{1}$. Ao atingir e ultrapassar essa fase, o exercício físico é considerado de intensidade moderada e intensa, pois ocorre aumento na concentração de ácido lático significantemente acima dos valores verificados no $L V_{1}$. As fibras musculares do tipo I aeróbias e a participação das fibras do tipo IIa e IIb de padrão glicolítico misto e anaeróbio, respectivamente, estimulam o aumento de ácido lático sanguíneo.

Nesse segundo instante metabólico, a resposta ventilatória é aumentada progressivamente, como tentativa de compensar a acidose metabólica. Além deste momento, a capacidade tampão do músculo é excedida e ocorre maior produção e acúmulo de ácido lático sanguíneo. A partir daí, se o exercício for mantido nessa condição fisiológica, por um longo período de tempo, o atleta será acometido de fadiga muscular intensa.

Portanto, o $\mathrm{LV}_{2}$ representa a intensidade de esforço acima da qual, durante um exercício de carga crescente, ocorre acúmulo de ácido lático no sangue e fadiga precoce.
No presente estudo, as atletas atingiram o $\mathrm{LV}_{2}$ a $86 \%$ do $\mathrm{VO}_{2}$ pico, com média de velocidade de $11,4 \mathrm{~km} \cdot \mathrm{h}^{-1}$. A média de resposta da FC verificada foi de $179 \mathrm{bpm}$, o que correspondeu a $93 \%$ da FC máxima atingida ao final do teste e a percepção subjetiva de cansaço, medida pela escala de Borg, atingiu o valor médio de 13 (ligeiramente cansativo).

Um $\mathrm{LV}_{2}$ elevado, ou seja, uma fração elevada do $\mathrm{VO}_{2}$ pico sem que haja acúmulo progressivo de ácido lático no sangue, tem importantes implicações funcionais. Basicamente, o atleta está melhor preparado para realizar atividades energéticas de maior intensidade por períodos de tempo mais prolongados. Conseqüentemente, é, sem dúvida, uma vantagem utilizar uma percentagem alta de seu $\mathrm{VO}_{2}$ pico sem entrar em acidose metabólica precoce.

É importante salientar que, em atletas, $\mathrm{o} \mathrm{LV}_{2}$ é fundamental quando se objetiva potencializar sua condição aeróbia, pois o exercício realizado nessa intensidade apresenta maior eficiência metabólica.

Os resultados demonstraram que a variabilidade dos limiares ventilatórios pode ocorrer em diferentes percentagens do $\mathrm{VO}_{2}$ pico para atletas com valor semelhante dessa variável ${ }^{16,17}$. Portanto, o exercício realizado a uma determinada intensidade pode representar graus variados de acidose metabólica em atletas com níveis de aptidão diferentes.

Essa condição pode ser uma fonte de variação importante em determinar a capacidade de um indivíduo tolerar exercício de longa duração.

Outro aspecto que valoriza esse índice fisiológico em atletas é que eles se exercitam mais próximos do $\mathrm{LV}_{2}$ do que no $\mathrm{LV}_{1}$, assemelhando-se ao esforço realizado durante as competições.

Outra importante qualidade física e o seu desenvolvimento é a potência aeróbia em futebolistas. Sabidamente, a solicitação física desse esporte é caracterizada por exercícios intermitentes e de longa duração.

Tradicionalmente, a potência aeróbia é conhecida como $\mathrm{VO}_{2}$ max. ou $\mathrm{VO}_{2}$ pico e tem sido aceita como um dos parâmetros fisiológicos, junto com $\mathrm{LV}_{2}$ e a economia de corrida, como os melhores indicadores da capacidade para o exercício prolongado ${ }^{18-20}$. Entretanto, alguns estudos ${ }^{21-23}$ demonstraram que em indivíduos saudáveis as diferenças genéticas contribuem, significativamente, para sua variabilidade.

Portanto, a modificação dessa variável metabólica, pelo treinamento, tem um limite biológico.

São poucos os estudos que relatam valores de $\mathrm{VO}_{2} \max$. ou $\mathrm{VO}_{2}$ pico em jogadoras de futebol. Recentemente, nosso laboratório de fisiologia do exercício publicou um estudo relatando valor médio de 47,3 $\pm 4,5 \mathrm{ml} \mathrm{O}{ }_{2} \cdot \mathrm{kg}^{-1} \cdot \mathrm{min}^{-1}$, encontrado em um grupo de jogadoras que se sagraram campeãs do II Campeonato Paulista de Futebol, em $1998^{24}$. Entretanto, há escassez significativa de estudos no futebol feminino, enfocando esse e outros parâmetros fisiológicos. 
Rhodes \& Mosher $^{25}$ verificaram, em 12 jogadoras universitárias canadenses de elite, valor médio de $47,1 \mathrm{ml} \mathrm{O}_{2} \cdot \mathrm{kg}^{-1}$. $\mathrm{min}^{-1}$. Resultado semelhante foi encontrado por Evangelista et al. $^{26}$, que verificaram em futebolistas italianas valor de $49,75 \mathrm{ml} \mathrm{O} \cdot \mathrm{kg}^{-1} \cdot \mathrm{min}^{-1}$.

Contudo, Jensen $\& \operatorname{Larsson}^{27}$ avaliaram jogadoras da seleção dinamarquesa e verificaram valor inicial levemente superior ao dos estudos acima citados, com valor médio de $53,3 \mathrm{ml}$ $\mathrm{O}_{2} \cdot \mathrm{kg}^{-1} \cdot \mathrm{min}^{-1}$. Após 15 semanas de treinamento, as mesmas atletas foram reavaliadas e o valor aumentou para $57,6 \mathrm{ml} \mathrm{O}_{2}$. $\mathrm{kg}^{-1} \cdot \mathrm{min}^{-1}$, um ganho de $8 \%$.

A importância de elevada potência aeróbia está relacionada com vários aspectos. Alguns estudos ${ }^{28-31}$ verificaram que níveis elevados de consumo de oxigênio exercem papel importante na recuperação mais rápida da energia proveniente do sistema fosfagênio (ATP-CP), responsável por considerável fornecimento de energia durante períodos de alta intensidade, como também atua na remoção mais eficiente do lactato em momentos de repouso ativo e/ou diminuição da intensidade do exercício.

Jacobs et al. ${ }^{32}$ afirmam que futebolistas masculinos, com potência aeróbia bem desenvolvida, produzem menos lactato em qualquer intensidade de exercício. O mesmo pensamento

\section{REFERÊNCIAS}

1. Kohno T, O'Hata N, Shirahata T, Hisatomi N, Endo Y. Physical characteristics of Japan women's national team soccer players. Abstract PJ11. FIMS Proceedings of World Congress of Sports Medicine. Amsterdam, May, 27-June 1, 1990:195.

2. Borg G. Perceived exertion as an indicator of somatic stress. Scan J Rehab Med 1970;2:92-6.

3. Bhambhani Y, Singh M. Ventilatory thresholds during a graded exercise test. Respiration 1985;47:120-8.

4. Glantz SA. Primer of biostatistics. 3rd ed. New York: McGraw-Hill, 1992.

5. Wasserman K, McIlroy MB. Detecting the threshold of anaerobic metabolism. Am J Cardiol 1964;14:844-52.

6. Ribeiro JP. Simpósio ABC - Cardiologia do Esporte. Limiares metabólicos e ventilatórios durante o exercício. Aspectos fisiológicos e metodológicos. Arq Bras Cardiol 1995;64:171-81.

7. Gomes PSC. Effects of continuous and intermittent training on body composition and selected physiological parameters. A thesis for the degree of doctor of philosophy. Edmonton, Alberta, 1989.

8. Hagberg JM, Coyle EF, Carroll JE, Miller JM, Martin WH, Brooke MH. Exercise hyperventilation in patients with McArdle's disease. J Appl Physiol 1982;52:991-4.

9. Reinhard U, Muller PH, Schmulling RM. Determination of anaerobic threshold by the ventilation equivalent in normal individuals. Respiration 1979;38:36-42.

10. Skinner JS, McLellan TM. The transition from aerobic to anaerobic metabolism. Res Q Exerc Sport 1980;51:234-48.

11. Saltin B, Gollnick P. Skeletal muscle adaptability: significance for metabolism and performance. In: Peache LD, Adrian R, Geiger SR, pode ser direcionado para as jogadoras de futebol, pois o que determina essa resposta fisiológica é o metabolismo aeróbio.

É importante salientar que a média de distância atingida ao final de uma partida, por jogadoras de futebol, é por volta de $8.500 \mathrm{~m}^{33}$. Além disso, elas realizam mais de 100 movimentos de sprint e sustentam uma resposta de freqüência cardíaca (FC) superior a $85 \%$ da FC máxima predita para a idade, por aproximadamente dois terços do jogo e mantendo intensidade relativa ao redor de $70 \%$ do consumo máximo de oxigênio.

Portanto, a potência aeróbia é uma das qualidade mais importantes a serem desenvolvidas em futebolistas femininas com o objetivo de suportar a longa duração das partidas.

Concluindo, a verificação de limiares ventilatórios e a potência aeróbia em jogadoras de futebol são parâmetros fisiológicos de grande importância, pois permitem expandir suas aplicações práticas, qualificando, controlando e desenvolvendo de modo mais adequado e objetivo o treinamento físico dessas atletas.

\section{AGRADECIMENTOS}

Agradecemos ao professor de língua inglesa Humberto Blancato pela correção gramatical do Abstract. editors. Handbook of physiology - Skeletal muscle. Baltimore: Williams and Wilkins, 1983:555-631.

12. Jorfeldt L. Metabolism of L (+) lactate in human skeletal muscle during exercise. Acta Physiol Scand 1970:Suppl 338.

13. Mader A, Liesen H, Heck H. Zur Berteilung der sportartspezifischen Ausdauerleistungsfahigkeit in labor. Sportarzt Sportmedizin 1976;27: 80-6.

14. Rusko H, Luhtanen P, Rahkila P, Viitasalo J, Rehunen S, Harkonen M. Muscle metabolism, blood lactate and oxygen uptake in steady state exercise at aerobic and anaerobic thresholds. Eur J Appl Physiol 1986; 55:181-6.

15. Casaburi R, Barstow TJ, Robinson T, Wasserman K. Dynamic and steady-state ventilatory and gas exchange responses to arm exercise. Med Sci Sports Exerc 1992;24:1365-74.

16. Davis JA, Frank MH, Whipp BJ, Wasserman K. Anaerobic threshold alterations caused by endurance training in middle-aged men. J Appl Physiol Respir Environ Exerc Physiol 1979;46:1039-46.

17. McLellan TM, Skinner JS. Blood lactate removal during active recovery related to the aerobic threshold. Int J Sports Med 1982;3:224-9.

18. Conley DL, Krahenbuhl GS. Running economy and distance running performance of highly trained athletes. Med Sci Sports Exerc 1980;12: 357-60.

19. Joyner MJ. Physiological limiting factors and distance running: influence of gender and age on record performance. Exerc Sports Sci Rev 1993;21:103-29.

20. Holmer I. Maximum oxygen uptake in athletes. J Appl Physiol 1967; 23:353-8.

21. Klissouras V. Prediction of athletic performance: genetic considerations. Can J Appl Sport Sci 1976;1:195-200. 
22. Bouchard C, Lortier G. Heredity and endurance performance. Sports Med 1984;1:38-64.

23. Bouchard C, Lortier G, Lesage R. Aerobic performance in brothers, dizygotic and monozygotic twins. Med Sci Sports Exerc 1986;18: $639-46$.

24. Silva PRS, Romano A, Andrade A, Riça WO, Cordeiro JR, et al. Características fisiológicas músculo-esqueléticas, antropométricas e oftalmológicas em jogadoras de futebol feminino consideradas de elite. Acta Fisiátrica 1997;5:18-26.

25. Rhodes EC, Mosher RE. Aerobic and anaerobic characteristics of elite female university soccer players. Communications to the Second World Congress of Science and Football. Eindoven, Nederlands 22-25, May, 1991. J Sports Sci (abstract) 1992;10:143.

26. Evangelista M, Pandolfi O, Fanton F, Faina M. A functional model of female soccer players: Analysis of functional characteristics. Communications to the Second World Congress of Science and Football. Eindoven, Nederlands 22-25, May, 1991. J Sports Sci (abstract) 1992;10: 165 .
27. Jensen K, Larsson B. Variations in physical capacity among the Danish national soccer team for women during a period of supplemental training. Communications to the Second World Congress of Science and Football. Eindoven, Nederlands 22-25, May, 1991. J Sports Sci (abstract) 1992;10:145.

28. Donovan CM, Brooks GA. Endurance training affects lactate clearance, not lactate production. Am J Physiol 1983;244:E83-E92.

29. Donovan CM, Pagliassotti MJ. Endurance training enhances lactate clearance during hyperlactatemia. Am J Physiol 1989;257:E782-E89.

30. Donovan CM, Pagliassotti MJ. Enhance efficiency of lactate removal after endurance training. J Appl Physiol 1990;68:1053-8.

31. MacRae HSH, Denis SC, Bosh NA, Noakes TD. Effects of training in lactate production and removal during progressive exercise in humans. J Appl Physiol 1992;72:1649-56.

32. Jacobs I, Westlin N, Karlsson J, Rasmousson M, Houghton B. Muscle glycogen and diet in elite soccer players. Eur J Appl Physiol 1982;48: 297-302.

33. Brewer J. Nutritional aspects of women's soccer. J Sports Sci 1994; 12:S35-S8 